This item was submitted to Loughborough's Research Repository by the author.

Items in Figshare are protected by copyright, with all rights reserved, unless otherwise indicated.

\title{
Emulsion copolymerization of styrene and butyl acrylate in the presence of a chain transfer agent. Part 2: parameters estimability and confidence regions
}

PLEASE CITE THE PUBLISHED VERSION

http://dx.doi.org/10.1016/j.ces.2012.12.013

\section{PUBLISHER}

(C) Elsevier

\section{VERSION}

AM (Accepted Manuscript)

\section{PUBLISHER STATEMENT}

This work is made available according to the conditions of the Creative Commons Attribution-NonCommercialNoDerivatives 4.0 International (CC BY-NC-ND 4.0) licence. Full details of this licence are available at: https://creativecommons.org/licenses/by-nc-nd/4.0/

\section{LICENCE}

CC BY-NC-ND 4.0

\section{REPOSITORY RECORD}

Benyahia, Brahim, M. Abderrazak Latifi, Christian Fonteix, and Fernand Pla. 2014. "Emulsion Copolymerization of Styrene and Butyl Acrylate in the Presence of a Chain Transfer Agent. Part 2: Parameters Estimability and Confidence Regions". figshare. https://hdl.handle.net/2134/15292. 


\title{
Emulsion copolymerization of styrene and butyl acrylate in the presence of a chain transfer agent. Part 2: Parameters estimability and confidence regions
}

\author{
B. Benyahia*, M. A. Latifi, C. Fonteix, F. Pla \\ Laboratoire Réactions et Génie des Procédés \\ CNRS-ENSIC,1 rue Grandville, BP 20451, 54001 Nancy Cedex, France
}

\begin{abstract}
Accurate estimation of the model parameters is required to obtain reliable predictions of the products end-use properties. However, due to the mathematical model structure and/or to a possible lack of measurements, the estimation of some parameters may be impossible. This paper will focus on the case where the main limitations to the parameters estimability are their weak effect on the measured outputs or the correlation between the effects of two or more parameters The objective of the method developed in this paper is to determine the subset of the most influencing parameters that can be estimated from the available experimental data, when the complete set of model parameters cannot be estimated. This approach has been applied to the mathematical model of the emulsion copolymerization of styrene and butyl acrylate, in the presence of n-dodecyl mercaptan as a chain transfer agent. In addition, a new approach is used to better assess the true confidence regions and evaluate the accuracy of the parameters estimates in more reliable way.
\end{abstract}

Keywords: emulsion copolymerization model, estimability analysis, parameter ranking, confidence regions.

\section{Introduction}

Building reliable mathematical models can be a very challenging task, particularly for complex systems which are commonly encountered in the fields of polymer engineering, system biology, and wastewater treatment. The common feature exhibited by this kind of models is the large number of parameters to be estimated. An accurate estimation of the whole set of parameters is usually impossible and the set is often reduced due to the insufficient information contained in the available experimental data or because the model structure.

\footnotetext{
* Current address : PSEL, MIT, 77 Massachusetts Ave., Cambridge MA 02139, USA.

Email address: benyahia@mit.edu (B. Benyahia)

${ }^{1}$ Preprint submitted to Chemical Engineering Science

${ }^{2}$ Please cite this article as:

Benyahia, B., Latifi, M. A., Fonteix, C., Pla, F., (2012). Chemical Engineering Science, 90, 110-118.
} 
A first step in the development of a reliable mathematical model, prior to the parameter identification problem, is to evaluate the structural identifiability and estimability (i.e. practical identifiability) of the model parameters. The first approach is exclusively based on the structure of the mathematical model and aims at investigating whether or not the model parameters are globally or locally structurally identifiable (Walter and Pronzato (1997), Quaiser and Mönnigmann (2009)). The estimability approach addresses a data problem. The objective is to determine the subset of parameters with the highest estimabililty potential, based on a predefined experimental design or available data. Due to the lack of measurements, accurate estimation of some parameters may be impossible. The main limitation to the parameters estimability is their weak influence on the measured outputs and/or the correlation between the parameters effects. Due to their poor accuracy, the estimation of these parameters can lead to significant degradation in the predictive capability of the model.

Different selection methods based on the estimability principle have been developed as reported in the literature, including the principal component method (Degenring et al. (2004), Turanyi (1990)), the singular value decomposition (Velez-Reyes and Verghese (1995)), the correlation methods (Jacquez and Greif (1985)) and the eigenvalue method (Vajda et al. (1989)). A good review of the most important methods can be found in Quaiser and Mönnigmann (2009) and McLean et al. (2011). The orthogonalization-based methods developed by Yao et al. (2003) and Lund and Foss (2008), are particularly efficient to rank the parameters with the highest estimability potential. Those methods have been widely used over the last five years in different research areas (Benyahia (2009), Jayasankar et al. (2009), Quiniou (2009), Ngo (2009), Surisetty et al. (2010)). In addition, the orthogonalization-based methods are relatively straightforward and can be used prior to the experimental campaign to identify the best parameter candidate to be estimated, based on the model predictions and an initial guess of the parameters vector, obtained generally from literature or previous identification procedure. When a parameter of interest is not estimable according the experimental design adopted, the latter may be modified to include sampling times corresponding to the highest influence of the parameter, which may potentially improve the estimability of this parameter if it is not correlated with another parameter.

In the first part of this two-part series, we presented the mathematical model of the emulsion copolymerization of styrene and butyl acrylate in the presence of a chain transfer agent. This model consisted of a system of differential algebraic equations (DAEs), derived from mass, population and moment equations (Benyahia et al. (2010)). In this paper, we implement the sequential orthogonalization method developed by Yao et al. (2003). Thanks to this approach, a subset of the most influential and linearly independent parameters is selected to the identification stage. On the other hand, it is highly desirable to assess the accuracy of the parameters estimates through the confidence intervals, which can be as important for the construction of a reliable model as the identification of the most likely parameters estimates.

The evaluation of uncertainties is key in process design, control, and optimization. The confidence regions provide a powerful tool to evaluate uncertainty. Traditionally, the confidence regions are obtained by methods based on the Fisher-Information-Matrix, under the assumption that the process model is linear in its parameters, and the experimental errors are Gaussian. This approach provides symmetric iso-contours (i.e., ellipsoids) centered by the parameters estimates. However, most of real systems exhibit high nonlinearities in parameters and show non-normal distribution errors. As a result, the elliptical confidence region may not be accurate enough to 
capture the actual model parameter or may underestimate the true confidence regions (Rooney and Biegler (2001)). The true confidence regions can be asymmetric, non-centered, unbounded, and even composed of unconnected parts depending on the model structure (Schwaab et al. (2008)). Different methods have been proposed in the literature to evaluate the true confidence regions starting by the Bootstrap and Monte-Carlo methods (Joshi et al. (2006)), (van Boekel (1996), Walter and Pronzato (1997)). Campi and Weyer (2005) proposed a method called the leave-out sign-dominant correlation regions. The method was applied to linear systems and assumes that the noise has zero mean without any restriction on the type of noise distribution (i.e., gaussian or non-gaussian) and the size of the data set. Varziri et al. (2008a) and Varziri et al. (2008b) used an approximate maximum likelihood estimation algorithm for parameter estimation in nonlinear dynamic models with model uncertainties and stochastic disturbances. They used the inverse of the Fisher information matrix as an approximation of the covariance matrix of the combined vector of states and parameters and derived a linearization-based expressions for the confidence regions. They also considered both cases: known and unknown variances of the disturbance intensities and measurement-noise. Schwaab et al. (2008) combined a particle swarm optimization procedure with the likelihood confidence region method to construct the true confidence regions for linear and non-linear systems. Yeh and Singh (1997) evaluated bootstrap confidence regions based on data depth, which is a geometric concept of ordering data from the center outwardly in higher dimensions. A good review of other methods can be found in Schwaab et al. (2008), Gallant (1987), and Seber and Wild (1989).

In this paper we present a new approach by witch the true confidence regions can be evaluated. The method is combined with the estimability analysis and provides a more reliable and robust approach to rank and select the model parameters. Indeed, monestimability can be identified by unrealistically large confidence intervals, while narrow ones indicate that the parameter estimate is accurate and consequently highly estimable. This statement supposes high accuracy in the evaluation of the confidence regions.

This paper is organized as follows: first the estimability method is described and discussed flowed by the description of the novel confidence region method. The two approaches are finally compared and discussed.

\section{Estimability analysis}

The process model developed in the first part of this series, describes a fed-batch process of the emulsion copolymerization of styrene and butyl acrylate in the presence of a chain transfer agent (Benyahia et al. (2010)). It was highly desirable to give a detailed and accurate description of underlying phenomena. The model describes different effects such as influence of the chain transfer agent on the polymerization kinetics and the glass and the gel effects. It also provides the population balance equations with respect to the number of radicals within the particles, and the equations of moments inherent to the macromolecules distributions. Although the number of the population balance equations has been reduced, the mathematical model is still large, complex, and nonlinear with respect to both states and parameters. The main challenge is to build a satisfactory predictable model with respect to the overall conversion $X_{\text {ove }}$, the residual mass fraction of styrene $F_{2}$, the number and weight average molecular weights $\left(\bar{M}_{n}, \bar{M}_{w}\right)$, and the average particles diameters $\left(d_{p}\right)$. Those outputs are closely related to the products end-use properties (both latex and polymers). As a consequence, any degradation in the model prediction capability may 
cause a poor control and/or optimization of the product quality.

The mathematical model which involves 49 parameters can be described by the general form of DAEs:

$$
\begin{aligned}
\dot{\boldsymbol{x}}(t) & =\boldsymbol{f}(\boldsymbol{x}(t), z(t), \boldsymbol{p}, t), \quad \boldsymbol{x}(t=0)=x_{0} \\
0 & =\boldsymbol{g}(\boldsymbol{x}(t), z(t), \boldsymbol{p}, t)
\end{aligned}
$$

where $\boldsymbol{x}$ is the vector of the differential state variables, $\boldsymbol{z}$ is the vector of the algebraic state variables, and $\boldsymbol{p}$ is the vector of the model parameters.

The nonlinear regression model has the following form:

$$
y_{i j}=\hat{y}_{i}\left(\boldsymbol{p}, t_{i j}\right)+\epsilon_{i j}
$$

where $y_{i j}$ the jth measurement of the ith output, $\hat{y}_{i}$ is the corresponding model predictions, $\epsilon_{i j}$ represents the data uncertainty (i.e,. noise) and $t_{i j}$ is the jth sampling time of the ith output.

Although noise can be in different forms such as human errors, random errors, unexpected disturbance, and measurements errors, only measurements errors are considered here and assumed to be uncorrelated, Gaussian distributed, additive, with zero mean.

The development of an effective solution to the parameter selection problem requires the quantification of the influence of each parameter on the measured outputs. This approach indicates which parameters are the most important and most likely to affect predictions of the model. The first step of the estimability analysis method is the evaluation of the sensitivity coefficients as follows,

$$
s_{i j}=\frac{\partial \hat{y}_{i}}{\partial p_{j}} \quad j=1,2, \ldots n_{p}
$$

where $n_{p}$ is the number of parameters.

The model parameters as well as the outputs have different units and span several orders of magnitude. A typical example of such case is the particle average diameter and the average molecular weights. To make a reliable comparison between the effects of the different parameters on the predictions of the model, the sensitivity coefficients are normalized with respect of the nominal values of the parameters and their corresponding output predicted by the mathematical model.

$$
\left.s_{i j}\right|_{t=t_{k}}=\left.\frac{\bar{p}_{j}}{\left.\bar{y}_{i}\right|_{t=t_{k}}} \frac{\partial \hat{y}_{i}}{\partial p_{j}}\right|_{t=t_{k}}
$$

where $\bar{p}_{j}$ is the nominal value of the jth parameter and $\left.\bar{y}_{i}\right|_{t=t_{k}}$ is the model prediction of the ith output evaluated at a sampling time $t_{k}$ using the nominal vector of the parameters $(\bar{p})$.

The overall sensitivities of the different outputs with respect to whole set of parameters, at different sampling times, can be summarized in a matrix of the sensitivity coefficients $(\boldsymbol{S})$. Each 
column of this matrix evaluates the global effect of a given parameter on the process outputs at different measurement times, whereas each row represents the effect of the whole set of parameters on a given output at a fixed time of measurement.

$$
S=\left[\begin{array}{lll}
\left.s_{11}\right|_{t=t_{1}} & \cdots & \left.s_{1 n_{p}}\right|_{t=t_{1}} \\
\vdots & \ddots & \vdots \\
\left.s_{n_{y} 1}\right|_{t=t_{1}} & \cdots & \left.s_{n_{y} n_{p}}\right|_{t=t_{1}} \\
\left.s_{11}\right|_{t=t_{2}} & \cdots & \left.s_{1 n_{p}}\right|_{t=t_{2}} \\
\vdots & \ddots & \vdots \\
\left.s_{n_{y} 1}\right|_{t=t_{n_{m}}} & \cdots & \left.s_{n_{y} n_{p}}\right|_{t=t_{n_{m}}}
\end{array}\right]
$$

If two columns of the matrix are linearly dependent, an accurate estimation of the corresponding parameters is not possible simultaneously, as the effect of one parameter can be modified or compensated by the effect of the other. Yao et al. (2003) used a modified Gram-Schmidt orthogonalization sequentially, to exclude the correlated parameters. In addition, the method provides an efficient way to distinguish the most influential parameters. When two parameters are correlated, the method makes it possible to select the one with the highest effect and exclude the other.

After the selection of the nominal values of the parameters, the sensitivity matrix can be computed analytically or by numerical approximation. The algorithm of the sequential orthogonalization estimability analysis is described as follows:

Let $\boldsymbol{s}_{i}$ be the sensitivity vector corresponding to the parameter $p_{i}, \boldsymbol{r}_{i}$ the orthogonal projection of $s_{i}, X_{j}$ the matrix of the selected parameters vectors at the jth stage, $\mathcal{P}$ the set of estimable parameters and $\lambda$ a cut-off value.

1. Selection of the parameter with the highest effect:

find the index $\mathrm{k}$ such that,

$$
\begin{gathered}
k=\arg \max _{i}\left(\boldsymbol{s}_{i}\right)^{T} \boldsymbol{s}_{i}, \quad i \in \boldsymbol{I}_{0}=\left\{1, \ldots, n_{p}\right\} \\
\text { if }\left(\boldsymbol{s}_{k}\right)^{T} \boldsymbol{s}_{k} \geq \lambda \quad \text { set } \mathcal{P}_{1}=\left\{p_{k}\right\} \text { and } X_{1}=\boldsymbol{s}_{k} \\
\text { otherwise stop }
\end{gathered}
$$

2. Orthogonalization:

compute the orthogonal projection of the matrix $S$,

$$
R^{j}=\left(I-X_{j}\left(X_{j}^{T} X_{j}\right)^{-1} X_{j}^{T}\right) S
$$

3. Selecting the next parameter with the highest effect:

$$
\begin{gathered}
l=\arg \max _{i}\left(\boldsymbol{r}_{i}^{j}\right)^{T} \boldsymbol{r}_{i}^{j}, i \in \boldsymbol{I}_{j}=\left(\boldsymbol{I}_{j-1}-\{k, \ldots\}\right) \\
\text { if }\left(\boldsymbol{r}_{l}^{j}\right)^{T} \boldsymbol{r}_{l}^{j} \geq \lambda \quad \begin{array}{c}
\text { set } \mathcal{P}_{j}=\left(\mathcal{P}_{j-1}, p_{l}\right) \text { and } X_{j+1}=\left(X_{j}, \boldsymbol{s}_{l}\right) \\
\text { return to step } 2 \\
\text { otherwise stop }
\end{array}
\end{gathered}
$$

After the selection of the first parameter, the vectors of the sensitivity matrix are projected sequentially on the space orthogonal to that spanned by the sensitivity vectors with the highest magnitude (i.e,. Euclidean norm). As a result, the vector selected in the previous step as well as 
Table 1: Nominal values of the parameters part 1.

\begin{tabular}{llll}
\hline Parameter & Value & Units & \multicolumn{1}{c}{ Reference } \\
\hline$E_{d}$ & 135000 & $\mathrm{~J} \cdot \mathrm{mol}^{-1}$ & Gilbert (1995) \\
$E_{p 11}$ & 22500 & $\mathrm{~J} \cdot \mathrm{mol}^{-1}$ & Ginsburger et al. (2003) \\
$E_{p 22}$ & 32500 & $\mathrm{~J} \cdot \mathrm{mol}^{-1}$ & Gilbert (1995) \\
$E_{t 22}$ & 9000 & $\mathrm{~J} \cdot \mathrm{mol}^{-1}$ & Sgard (2000) \\
$E_{t r m 11}$ & 20000 & $\mathrm{~J} \cdot \mathrm{mol}^{-1}$ & Ginsburger et al. (2003) \\
$m_{d 1}, m_{d 2}$ & 39 & & Rawlings and Ray (1988) \\
$n_{s}$ & 40 & & Ginsburger et al. (2003) \\
$E_{e}$ & 361 & $\mathrm{~K}$ & Ginsburger et al. (2003) \\
$\delta_{m 1}, \delta_{m 2}$ & 0.03 & & Ginsburger et al. (2003) \\
$\delta_{1}$ & 31 & & Ginsburger et al. (2003) \\
$\delta_{2}$ & 22 & & Ginsburger et al. (2003) \\
$K_{p 1}$ & $1 / 1050$ & & Gugliotta et al. (1995) \\
$K_{p 2}$ & $1 / 2512$ & & Gugliotta et al. (1995) \\
$r_{m i c}$ & 2.5 & $n m$ & Gilbert (1995) \\
$r_{d}$ & 5000 & $n m$ & Gilbert (1995) \\
$D_{w 1}, D_{w 2}$ & $4.1 \cdot 10^{-7}$ & $\mathrm{~m}^{2} \cdot \mathrm{s}^{-1}$ & Arzamendi et al. (1992) \\
$a_{11}^{g l}$ & 17.13 & & Martinet (1992) \\
$a_{12}^{g l}, a_{21}^{g l}, a_{22}^{g l}$ & 5.73 & & Martinet (1992) \\
$b^{g e}$ & 11.46 & & Martinet (1992) \\
$b^{g l}$ & 3.78 & & Martinet (1992) \\
$a_{e}$ & 0.75 & $n m^{2}$ & Ginsburger et al. (2003) \\
$k_{T A, d w} A_{d}$ & $5 / 6$ & $\mathrm{~m}^{3} \cdot \mathrm{s}^{-1}$ & Salazar et al. (1998) \\
$\tau$ & $2 / 3$ & & Ginsburger et al. (2003) \\
\hline & & &
\end{tabular}

all its linearly dependent vectors, will have a null vector as projection, and will have no influence on the subsequent selection procedure which is based on the highest magnitude. This method will consequently guarantee that the next selected vector will have the least correlation possible with the previously selected parameters while exhibiting the highest relative effect on the measurements.

The estimability method presented here, is however a local approach which requires a good initial guess of the parameters vector. Unrealistic or highly inaccurate initial parameter values can lead to unreliable parameter ranking which may cause a significant degradation of the model prediction capabilities. Most of the parameter values used in the estimablity are obtained from literature, particularly from similar systems as summarized in tables 1 and 2 . Some of the parameters whose values are unknown or for which literature provides poor initial guesses required a pre-identification. This is the case of the parameters related the chain transfer agent: $E_{T A p 1}$, $E_{T A p 2}, K_{p t a}, k_{T A p 10}$, and $k_{T A p 20}$. The identification procedure used is the maximum likelihood approach in a global optimization framework. It required the use of the data available and the nominal values of the remaining parameters. The reliability of this approach assumes that the parameters at hand are poorly correlated. 
Table 2: Nominal value of the parameters part 2 .

\begin{tabular}{llll}
\hline Parameter & Value & Units & Reference \\
\hline$E_{T A p 1}$ & 78800 & $\mathrm{Kj} / \mathrm{kmol}$ & Optimized \\
$E_{T A p 2}$ & 36200 & $\mathrm{Kj} / \mathrm{kmol}$ & Optimized \\
$E_{t 11}$ & 11000 & $\mathrm{Kj} / \mathrm{kmol}$ & Ginsburger et al. (2003) \\
$E_{t r m 22}$ & 40000 & $\mathrm{KJ} / \mathrm{kmol}$ & Ginsburger et al. (2003) \\
$f_{0}$ & 0.92 & & Ginsburger et al. (2003) \\
$K_{p t a}$ & 0.4 & & Optimized \\
$K_{p Z}$ & 0.4 & & Ginsburger et al. (2003) \\
$k_{d 0}$ & $10^{-6}$ & $\mathrm{~s}^{-1}$ & Ginsburger et al. (2003) \\
$k_{p 110}$ & 190 & $\mathrm{~m}^{3} \cdot \mathrm{kmol}^{-1} \cdot \mathrm{s}^{-1}$ & Ginsburger et al. (2003) \\
$k_{p 220}$ & 34.9 & $\mathrm{~m}^{3} \cdot \mathrm{kmol}^{-1} \cdot \mathrm{s}^{-1}$ & Ginsburger et al. (2003) \\
$k_{T A p 10}$ & 515 & $\mathrm{~m}^{3} \cdot \mathrm{kmol}^{-1} \cdot \mathrm{s}^{-1}$ & Optimized \\
$k_{T A p 20}$ & 17.8 & $\mathrm{~m}^{3} \cdot \mathrm{kmol}^{-1} \cdot \mathrm{s}^{-1}$ & Optimized \\
$k_{t 110}$ & $6 \cdot 10^{9}$ & $\mathrm{~m}^{3} \cdot \mathrm{kmol}^{-1} \cdot \mathrm{s}^{-1}$ & Ginsburger et al. (2003) \\
$k_{t 220}$ & $7 \cdot 10^{9}$ & $\mathrm{~m}^{3} \cdot \mathrm{kmol}^{-1} \cdot \mathrm{s}^{-1}$ & Ginsburger et al. (2003) \\
$k_{t r m 110}$ & 0.005 & $\mathrm{~m}^{3} \cdot \mathrm{kmol}^{-1} \cdot \mathrm{s}^{-1}$ & Ginsburger et al. (2003) \\
$k_{t r m 220}$ & 0.011 & $\mathrm{~m}^{3} \cdot \mathrm{kmol}^{-1} \cdot \mathrm{s}^{-1}$ & Ginsburger et al. (2003) \\
$k_{z p}$ & 100 & $\mathrm{~m}^{3} \cdot \mathrm{kmol}^{-1} \cdot \mathrm{s}^{-1}$ & Ginsburger et al. (2003) \\
$r_{p 21}$ & 0.65 & & Snuparek and Kleckova (1984) \\
$r_{p 12}$ & 0.25 & & Snuparek and Kleckova (1984) \\
$\epsilon$ & 11.74 & $\mathrm{~m}$ & Ginsburger et al. (2003) \\
$\sigma$ & 1.3 & & Arzamendi et al. (1992) \\
\hline
\end{tabular}

\begin{tabular}{|c|c|c|c|c|c|c|c|c|}
\hline Cut-off & $\mathcal{P}$ & & & & & & & \\
\hline 21 & $\sigma$ & & & & & & & \\
\hline 15 & $\sigma$ & $f_{0}$ & & & & & & \\
\hline 12 & $\sigma$ & $f_{0}$ & $k_{d 0}$ & & & & & \\
\hline 10 & $\sigma$ & $f_{0}$ & $k_{d 0}$ & $r_{p 21}$ & & & & \\
\hline 1.8 & $\sigma$ & $f_{0}$ & $k_{d 0}$ & $r_{p 21}$ & $E_{T A p 1}$ & & & \\
\hline 1.3 & $\sigma$ & $f_{0}$ & $k_{d 0}$ & $r_{p 21}$ & $E_{T A p 1}$ & $k_{p 220}$ & & \\
\hline 0.72 & $\sigma$ & $f_{0}$ & $k_{d 0}$ & $r_{p 21}$ & $E_{T A p 1}$ & $k_{p 220}$ & $K_{p t a}$ & \\
\hline : & $\vdots$ & & : & & & & & \\
\hline 0.01 & $\sigma$ & $f_{0}$ & $k_{d 0}$ & $r_{p 21}$ & $E_{T A p 1}$ & $k_{p 220}$ & $K_{p t a}$ & $\ldots \quad k_{t 220}$ \\
\hline
\end{tabular}

Table 3: Subsets of the most estimable parameters obtained for different cut-off values. 


\begin{tabular}{|ll|ll|ll|}
\hline Parameter & rank & Parameter & rank & Parameter & rank \\
\hline$\sigma$ & 1 & $K_{p t a}$ & 8 & $k_{t r m 110}$ & 15 \\
$f_{0}$ & 2 & $k_{T A p 20}$ & 9 & $k_{z p}$ & 16 \\
$k_{d 0}$ & 3 & $k_{t r m 220}$ & 10 & $E_{t 11}$ & 17 \\
$r_{p 21}$ & 4 & $E_{t r m 22}$ & 11 & $k_{t 110}$ & 18 \\
$r_{p 12}$ & 5 & $k_{T A p 10}$ & 12 & $K_{p Z}$ & 19 \\
$E_{T A p 1}$ & 6 & $k_{p 110}$ & 13 & $\epsilon$ & 20 \\
$k_{p 220}$ & 7 & $E_{T A p 2}$ & 14 & $k_{t 220}$ & 21 \\
\hline
\end{tabular}

Table 4: Ranking of the parameters with the highest estimability potential.

Different parameters subsets can be obtained according to the cut-off values imposed, as shown in table 3 . The order of appearance gives the priority with respect to the estimability potential. The orthogonalization method used in this work provides 21 parameters selected among the 49 model parameters, Those parameters are ranked in table 4. Although the cut-off value is of paramount importance, a unique and efficient approach to select its value is not reported in the literature. Instead, the cut-off value can be specified arbitrarily a priori (Yao et al. (2003)) or refined with respect to the value of the objective function for the parameter estimation (Wu et al. (2011), Littlejohns et al. (2010)).

The effect of the cut-off value on the number of selected parameters is depicted in Figure 1. This seems to indicate that a number of parameters equal to $10 \mathrm{might}$ be sufficient. The practice shows that the objective function (maximum likelihood criterion) and consequently the agreement between the model predictions and the measurements, can be further improved by selecting more parameters. A satisfactory trade-off between a minimum number of parameters and high accuracy of the model prediction, is finally met with 21 parameters ranked in table 4. An alternative approach to further improve the accuracy of the model might be obtained by setting new experiments. However, the approach presented in this paper was restricted to the experimental data available which gave satisfactory results.

The 21 parameters estimates were obtained by minimizing the maximum likelihood criterion as explained in detail, in the first part of this series (Benyahia et al. (2010)).

$$
\begin{aligned}
& \operatorname{Min} J(\boldsymbol{p})= \sum_{i=1}^{n_{y}} n_{m i} \cdot \ln \left(\sum_{j=1}^{n_{m i}}\left(y_{i j}-\hat{y}_{i}\left(\boldsymbol{x}, \boldsymbol{p}, t_{i j}\right)\right)^{2}\right) \\
& \text { s.t } \quad \boldsymbol{x}=\boldsymbol{f}(\boldsymbol{x}(t), \boldsymbol{z}(t), \boldsymbol{u}(t), \boldsymbol{p}, t) \quad \boldsymbol{x}(t=0)=\boldsymbol{x}_{0} \\
& 0=\boldsymbol{g}(\boldsymbol{x}(t), \boldsymbol{z}(t), \boldsymbol{p}, t) \\
& \boldsymbol{p}_{\text {inf }} \leq \boldsymbol{p} \leq \boldsymbol{p}_{\text {sup }} \\
& \boldsymbol{p}^{T}=\left[\sigma, f_{0}, k_{d 0}, r_{p 21}, r_{p 12}, E_{T A p 1}, k_{p 220}, K_{p t a}, k_{T A p 20},\right. \\
& k_{t r m 220}, E_{t r m 22}, k_{T A p 10}, k_{p 110}, E_{T A p 2}, k_{t r m 110}, k_{z p}, \\
&\left.E_{t 11}, k_{t 110}, K_{p Z}, \epsilon, k_{t 220}\right]
\end{aligned}
$$

After the parameters identification, the mathematical model undergone successfully series 
of validation under batch and fed-batch conditions. In addition, this model was used for the multiobjective optimization of the process and showed again satisfactory prediction capabilities, particularly when the optimal feeding profiles were implemented experimentally (Benyahia et al. (2011)).

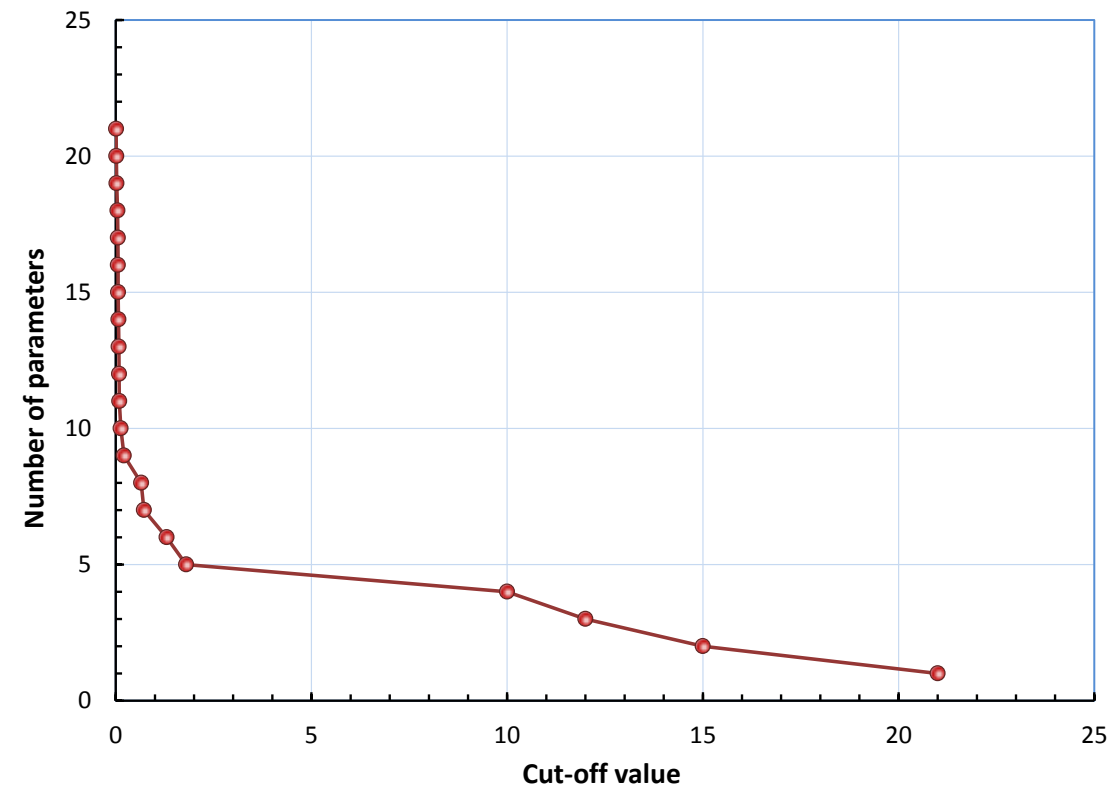

Figure 1: Number of selected parameters vs. cut-off value.

\section{Confidence regions}

The confidence regions provide a useful tool to evaluate the accuracy of the parameter estimates. However, due to poor evaluations of the true confidence regions, the accuracy may be overestimated which may alter the reliability of a mathematical model. The main limitation to an accurate evaluation of the true confidence region is the high nonlinearity exhibited by most real systems with respect to the parameters, and a non-normal distribution of the measurements errors, particularly when the number of measurement is too low. Here we will describe an alternative to the traditional ellipsoidal centered (asymptotic) confidence regions. 


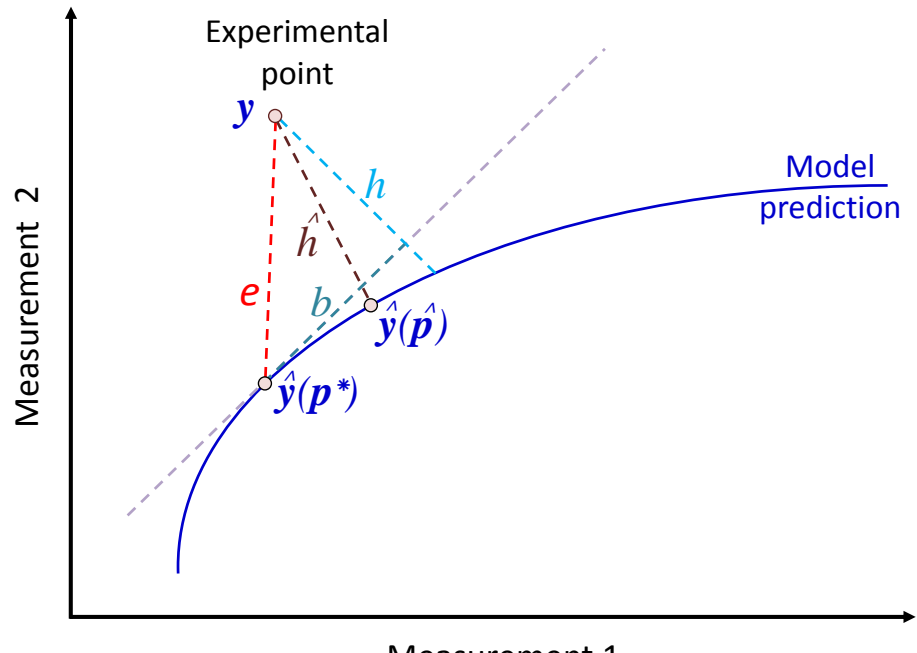

Measurement 1

Figure 2: Simplified representation of the experimental measurements and the model predictions in the parameters space.

Figure 2 represents the model prediction in the measurements space. In this case: $e$ is the difference between the experimental measurements $(\mathbf{y})$ and the model predictions based on the vector of the true values of the parameters $\left(\hat{\mathbf{y}}\left(\boldsymbol{p}^{*}\right)\right), b$ is the projection of $e$ on a plan tangent to the model predictions at $\hat{\mathbf{y}}\left(\boldsymbol{p}^{*}\right), \hat{h}$ is the difference between the experimental measurements and the model prediction based on the vector of the parameter estimates $(\hat{\boldsymbol{y}}(\hat{\boldsymbol{p}}))$ and $h$ is the vertical distance between the experimental measurements and the tangent plan.

The error $e$ can be expressed as,

$$
e^{2}=b^{2}+h^{2}
$$

The square error between the experimental value and the model prediction of the jth measurement of the ith output is given by,

$$
\epsilon_{i j}^{2}=\left(y_{i j}-\hat{y}_{i}\left(\boldsymbol{p}^{*}, t_{i j}\right)\right)^{2}
$$

This error can be normalized with respect to the variance as follows:

$$
e_{i j}^{2}=\frac{\left(y_{i j}-\hat{y}_{i}\left(\boldsymbol{p}^{*}, t_{i j}\right)\right)^{2}}{V_{i}}
$$


where $V_{i}$ is the true variance of the ith output measurements.

The previous approach can be generalized to all measurements of the ith output and for all outputs,

$$
\begin{aligned}
e_{i}^{2} & =\sum_{j=1}^{n_{m i}} \epsilon_{i j}^{2}=\sum_{j=1}^{n_{m i}} \frac{\left(y_{i j}-\hat{y}_{i}\left(\boldsymbol{p}^{*}, t_{i j}\right)\right)^{2}}{V_{i}} \\
e^{2} & =\sum_{i=1}^{n_{y}} e_{i}^{2}=\sum_{i=1}^{n_{y}} \sum_{j=1}^{n_{m i}} \frac{\left(y_{i j}-\hat{y}_{i}\left(\boldsymbol{p}^{*}, t_{i j}\right)\right)^{2}}{V_{i}}
\end{aligned}
$$

where $n_{m i}$ is the total number of measurements of the ith output and $n_{y}$ the total number of outputs.

The overall errors between experimental measurements and the model predictions based on the parameter estimates, can be obtained in a similar way.

$$
\begin{aligned}
& \hat{h}_{i}^{2}=\sum_{j=1}^{n_{m i}} \hat{h}_{i j}^{2} \\
& \hat{h}^{2}=\sum_{i=1}^{n_{y}} \hat{h}_{i}^{2}
\end{aligned}
$$

The variance of the ith output evaluated with respect to the parameter estimates $\left(\hat{V}_{i}(\hat{\boldsymbol{p}})\right)$ is obtained by,

$$
\begin{gathered}
\hat{V}_{i}=\frac{1}{n_{m i}} \sum_{j=1}^{n_{m i}}\left(y_{i j}-\hat{y}_{i}\left(\hat{\boldsymbol{p}}, t_{i j}\right)\right)^{2} \\
=\frac{1}{n_{m i}} \sum_{j=1}^{n_{m i}} \hat{h}_{i j}^{2} V_{i}
\end{gathered}
$$

thus

$$
\hat{h}_{i}^{2}=\frac{n_{m i} \hat{V}_{i}}{V_{i}}
$$

In a similar way, the variance of the ith output evaluated with respect to the vector of the true parameter $\left(\tilde{V}_{i}\left(\boldsymbol{p}^{*}\right)\right)$ gives,

$$
e_{i}^{2}=\frac{n_{m i} \tilde{V}_{i}\left(\boldsymbol{p}^{*}\right)}{V_{i}}
$$

The errors $e$ and $\hat{h}$ are both supposed Gaussian distributed with zero mean. This assumption is not unrealistic, particularly when the number of measurements is large. The central limit theorem provides us with the proof that even if the small sized density is not normal, it tends to be normal when the number of elements increases asymptotically to infinity. As a consequence the square errors have chi square distribution, 


$$
\begin{aligned}
& e^{2} \rightarrow \chi^{2}\left(n_{m}\right) \\
& \hat{h}^{2} \rightarrow \chi^{2}\left(n_{m}-n_{p}-n_{y}+1\right)
\end{aligned}
$$

where $\chi^{2}$ is chi square distribution, $n_{m}$ is the total number of measurements and $n_{p}$ the total number of parameters.

There are $n_{m}$ (independent) measurements. The error $e^{2}$ is therefore a chi square distribution with $n_{m}$ degrees of freedom. On the other hand, $\hat{h}^{2}$ is the result of an optimization where the derivatives of the probability density with respect to the $n_{m}$ parameters and the $n_{y}$ variances are set equal to zero. In addition, one variance can be used as a reference. As a result, $\hat{h}^{2}$ is a chi square distribution with $n_{m}-\left(n_{p}+n_{y}-1\right)$ degrees of freedom.

The difference $\left(e^{2}-\hat{h}^{2}\right)$ gives a chi square distribution with $n_{m}-\left(n_{m}-n_{p}-n_{y}+1\right)$ degrees of freedom,

$$
e^{2}-\hat{h}^{2} \rightarrow \chi^{2}\left(n_{p}+n_{y}-1\right)
$$

On the other hand, the following approximation is used.

$$
\frac{b^{2}}{h^{2}}=\frac{e^{2}-h^{2}}{h^{2}} \cong \frac{e^{2}-\hat{h}^{2}}{\hat{h}^{2}}
$$

$b$ and $h$ are independent (i.e,. orthogonal). As a ratio of two independent chi square distribution is a Fisher-Snedecor distribution, we obtain,

$$
\begin{aligned}
& \frac{\frac{e^{2}-\hat{h}^{2}}{n_{p}+n_{y}-1}}{\hat{h}^{2}} \rightarrow F_{\alpha}\left(n_{p}+n_{y}-1, n_{m}-n_{p}-n_{y}+1\right) \\
& \overline{n_{m}-n_{p}-n_{y}+1} \\
& \frac{\sum_{i=1}^{n_{y}} \frac{n_{m i} \tilde{V}_{i}\left(\boldsymbol{p}^{*}\right)}{V_{i}}-\sum_{i=1}^{n_{y}} \frac{n_{m i} \hat{V}_{i}}{V_{i}}}{\sum_{i=1}^{n_{y}} \frac{n_{m i} \hat{V}_{i}}{V_{i}}} \rightarrow \frac{n_{p}+n_{y}-1}{n_{m}-n_{p}-n_{y}+1} F_{\alpha}\left(n_{p}+n_{y}-1, n_{m}-n_{p}-n_{y}+1\right)
\end{aligned}
$$

where $\alpha$ is the level of confidence, $\left(n_{p}+n_{y}-1\right)$ and $\left(n_{m}-n_{p}-n_{y}+1\right)$ the degrees of freedom.

To evaluate the confidence regions with the Fisher-Snedecor test. Equation 19 will be expressed in terms of maximum likelihood criterion with respect to the vector of the true parameters and the vector of the parameter estimates,

$$
J\left(\boldsymbol{p}^{*}\right)=\sum_{i=1}^{n_{y}} n_{m i} \cdot \ln \left(n_{m i} \tilde{V}_{i}\left(\boldsymbol{p}^{*}\right)\right)
$$


By adding the term $-V_{i}+V_{i}$, the maximum likelihood leads to,

$$
\begin{aligned}
J\left(\boldsymbol{p}^{*}\right) & =\sum_{i=1}^{n_{y}} n_{m i} \cdot \ln \left(n_{m i}\left(\tilde{V}_{i}\left(\boldsymbol{p}^{*}\right)-V_{i}+V_{i}\right)\right) \\
& =\sum_{i=1}^{n_{y}} n_{m i} \cdot \ln \left(n_{m i} V_{i}\right)+\sum_{i=1}^{n_{y}} n_{m i} \cdot \ln \left(1+\frac{\tilde{V}_{i}\left(\boldsymbol{p}^{*}\right)-V_{i}}{V_{i}}\right)
\end{aligned}
$$

The term $\frac{\tilde{V}_{i}(\boldsymbol{p})-V_{i}}{V_{i}}$ is expected to be very low compared to 1 . As a result,

$$
\begin{aligned}
J\left(\boldsymbol{p}^{*}\right) & \approx \sum_{i=1}^{n_{y}} n_{m i} \cdot \ln \left(n_{m i} V_{i}\right)+\sum_{i=1}^{n_{y}} n_{m i} \cdot\left(\frac{\tilde{V}_{i}\left(\boldsymbol{p}^{*}\right)-V_{i}}{V_{i}}\right) \\
& =\sum_{i=1}^{n_{y}} n_{m i} \cdot \ln \left(n_{m i} V_{i}\right)-n_{m}+\sum_{i=1}^{n_{y}} n_{m i} \cdot\left(\frac{\tilde{V}_{i}\left(\boldsymbol{p}^{*}\right)}{V_{i}}\right)
\end{aligned}
$$

The equation 22 is rearranged as fiollows:

$$
\sum_{i=1}^{n_{y}} \frac{n_{m i} \tilde{V}_{i}\left(\boldsymbol{p}^{*}\right)}{V_{i}}=J\left(\boldsymbol{p}^{*}\right)-\sum_{i=1}^{n_{y}} n_{m i} \cdot \ln \left(n_{m i} V_{i}\right)+n_{m}
$$

Similarly the maximum likelihood criterion with respect to vector of the parameter estimates is given by,

$$
\sum_{i=1}^{n_{y}} \frac{n_{m i} \tilde{V}_{i}}{V_{i}}=J(\hat{\boldsymbol{p}})-\sum_{i=1}^{n_{y}} n_{m i} \cdot \ln \left(n_{m i} V_{i}\right)+n_{m}
$$

By substituting equations 23 and 24 in equation 19, we get,

$$
\frac{J\left(\boldsymbol{p}^{*}\right)-J(\hat{\boldsymbol{p}})}{\sum_{i=1}^{n_{y}} \frac{n_{m i} \hat{V}_{i}}{V_{i}}} \leq \frac{n_{p}+n_{y}-1}{n_{m}-n_{p}-n_{y}+1} F_{\alpha}\left(n_{p}+n_{y}-1, n_{m}-n_{p}-n_{y}+1\right)
$$

Finally, by assuming that $\hat{V}_{i}$ provides a good approximation to $V_{i}\left(\hat{V}_{i} \approx V_{i}\right)$, equation 19 reduces to,

$$
J\left(\boldsymbol{p}^{*}\right)-J(\hat{\boldsymbol{p}}) \leq \frac{n_{m}\left(n_{p}+n_{y}-1\right)}{n_{m}-n_{p}-n_{y}+1} F_{\alpha}\left(n_{p}+n_{y}-1, n_{m}-n_{p}-n_{y}+1\right)
$$

or

$$
J\left(\boldsymbol{p}^{*}\right) \leq J(\hat{\boldsymbol{p}})+\frac{n_{m}\left(n_{p}+n_{y}-1\right)}{n_{m}-n_{p}-n_{y}+1} F_{\alpha}\left(n_{p}+n_{y}-1, n_{m}-n_{p}-n_{y}+1\right)
$$

The Fisher-Snedecor test presented in equation 26 provides a statistical test to assess the parameter reliability. It indicates that the true parameters values are located within the confidence regions at a given probability. Schwaab et al. (2008) derived an expression based on FisherSnedecor test, where the objective function was expressed in terms of least-squares function and 
represent. the extension of the test derived by Beale (1960) to the nonlinear case.

The confidence regions are evaluated for a level of confidence equal to $90 \%(\alpha=0.1)$, the number of measurment $n_{m}$ is 161 (see Benyahia et al. (2010) for more detail about the number of experiments and measurment), the number of outputs $n_{y}$ is 5 and the number of parameters identified $n_{p}$ is 21 . In addition, the test requires the value of the maximum likelihood criterion at the optimum, $J(\hat{\boldsymbol{p}})=75.81$.

A satisfactory construction of the confidence regions requires the evaluation of a very large number of points to select those satisfying Fisher-Snedecor test (equation 26). The heuristic optimization methods, such as simulated annealing and particle swarm optimization and genetic algorithms provide an efficient and easy way to address this problem (Schwaab et al. (2008)). Those methods have the capability for solving high-dimensional, non-smooth, discontinuous, and multi-modal problems easily compared to the gradient based methods. A genetic algorithm is consequently used to construct the confidence regions. This algorithm can be used to evaluate simultaneously the parameter estimate and their confidence regions. As the number of parameters at hand is large, only a sample of joint confidence regions is presented in the paper, for the sake of brevity. Nevertheless, the examples presented in figure 3 and 4 , show parameters top ranked (e.g. $\sigma, k_{d 0}, k_{d 0}$ ), from the middle (e.g. $\left.k_{t r m 220}, E_{t r m 22}\right)$ and from the bottom (e.g. $K_{p Z}$, $\left.\epsilon, k_{t 220}\right)$. This will provide an overview on the accuracy at different levels of the estimability potential.

The confidence regions obtained by clusters of points in figures 3: A, B, and C, are ellipsoidal and centered. However, the confidence regions depicted in figure 3: D and figures 3: C and D, are asymmetric and non-centered. This is typically inherent to nonlinearities with respect to the parameters. In addition, the confidence regions depicted in figures 3: A, B, C, and figures 4: A, $\mathrm{B}$, show that the parameters at hand, are not correlated as the confidence regions are not inclined. However, no conclusion can be made for the remaining parameters with asymmetric confidence regions. The evaluation of their correlation degree requires in this case, the calculation of their correlation coefficients.

The confidence intervals of the 21 parameters are obtained by the projection of the confidence region onto each parameter axis. In addition, the interval bounds are normalized with respect to the optimal values of the parameters (i.e,. the parameter estimates) to evaluate their relative accuracy (table 5). A narrow confidence interval indicates higher accuracy in the parameter estimate. This also means that a small change in the parameter value has a large effect on the objective function, which in turn indicates a large effect on the output predictions. On the other hand, unrealistically large confidence intervals are typically the consequence of a weak effect of the parameter at hand, on the process outputs.

The normalized confidence intervals are represented in figure 5, to provide an efficient comparison between the relative accuracy of the 21 parameters. This overview reveals that the top ranked parameters, particularly $\sigma, k_{d 0}, r_{p 12}$, and $E_{T A p 1}$, have the highest relative accuracy, whilst the last parameters (e.g. $k_{t r m 110}, k_{z p}$, and $K_{p Z}$ ) have the lowest. This indicates a good agreement between the parameters outranking based on the estimability analysis, and the relative accuracy of their estimates. However, the estimability analysis is a local approach and discrepancies between the two approaches are likely to occur. This is for instance the case of the sixth $E_{T A p 1}$ for 

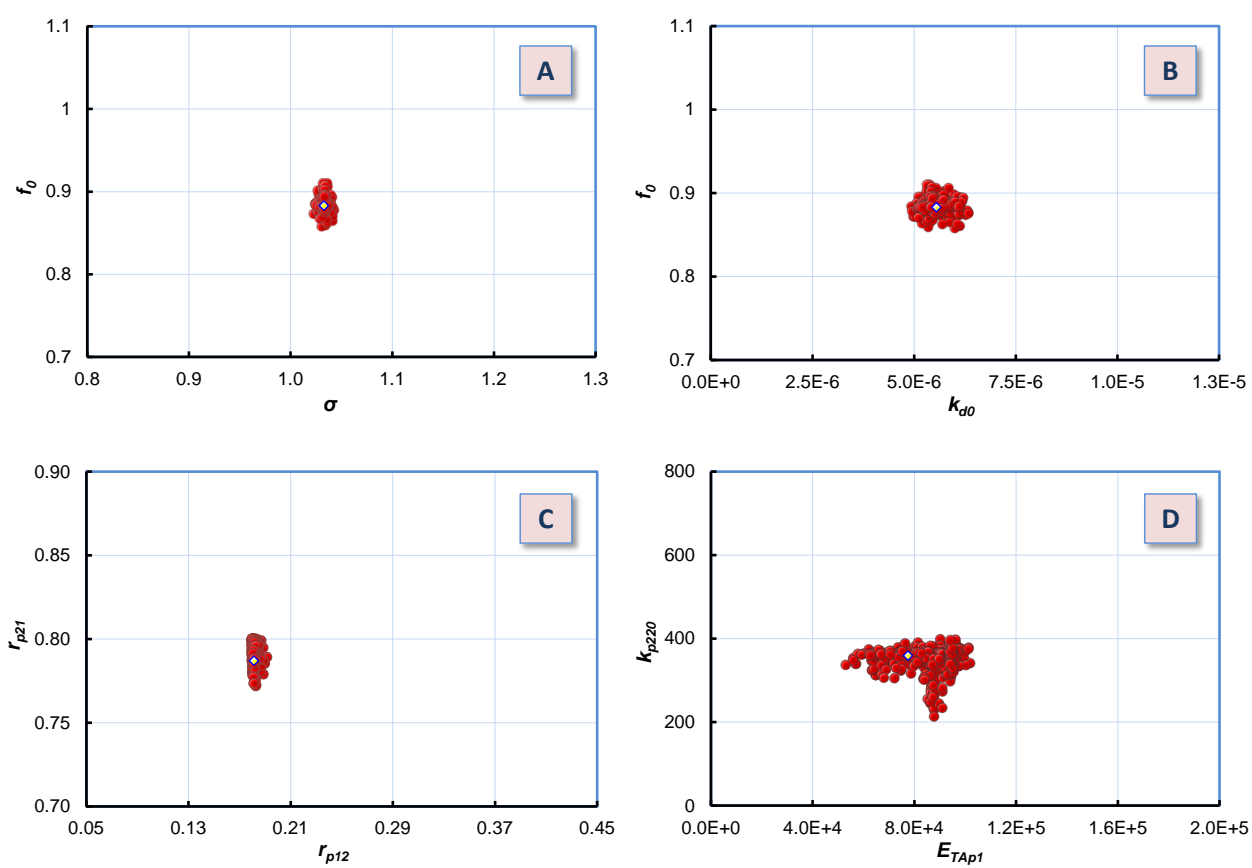

Figure 3: Joint confidence regions of A. $f_{0}$ (a parameter related to the initiator efficiency) vs. $\sigma$ (swelling parameters of the particles), B. $f_{0}$ vs. $k_{d 0}$ (kinetic coefficient of the initiator decomposition), C. $r_{p 21}$ (reactivity ratio of styrene) vs. $r_{p 12}$ (reactivity ratio of butyl acrylate), D. $k_{p 220}$ (propagation rate coefficient of styrene) vs. $E_{T A p 1}$ (activation energy of transfer to butyle acrylate)

\begin{tabular}{|llc||llc|}
\hline rank & Parameter & $\begin{array}{c}\text { Confidence } \\
\text { interval }\end{array}$ & rank & Parameter & $\begin{array}{c}\text { Confidence } \\
\text { interval }\end{array}$ \\
\hline 1 & $\sigma$ & {$[0.993,1.013]$} & 12 & $k_{T A p 10}$ & {$[0.489,1.383]$} \\
2 & $f_{0}$ & {$[0.997,1.034]$} & 13 & $k_{p 110}$ & {$[0.573,1.259]$} \\
3 & $k_{d 0}$ & {$[0.896,1.153]$} & 14 & $E_{T A p 2}$ & {$[0.676,1.353]$} \\
4 & $r_{p 21}$ & {$[0.987,1.026]$} & 15 & $k_{t r m 110}$ & {$[0.480,1.900]$} \\
5 & $r_{p 12}$ & {$[0.983,1.056]$} & 16 & $k_{z p}$ & {$[0.227,1.800]$} \\
6 & $E_{T A p 1}$ & {$[0.841,1.619]$} & 17 & $E_{t 11}$ & {$[0.446,1.154]$} \\
7 & $k_{p 220}$ & {$[0.593,1.139]$} & 18 & $k_{t 110}$ & {$[0.371,1.126]$} \\
8 & $K_{p t a}$ & {$[0.909,1.200]$} & 19 & $K_{p Z}$ & {$[0.282,2.470]$} \\
9 & $k_{T A p 20}$ & {$[0.487,1.174]$} & 20 & $\epsilon$ & {$[0.669,1.673]$} \\
10 & $k_{t r m 220}$ & {$[0.667,1.222]$} & 21 & $k_{t 220}$ & {$[0.254,1.513]$} \\
11 & $E_{t r m 22}$ & {$[0.908,1.124]$} & & & \\
\hline
\end{tabular}

Table 5: Normalized confidence intervals of the 21 parameters. 

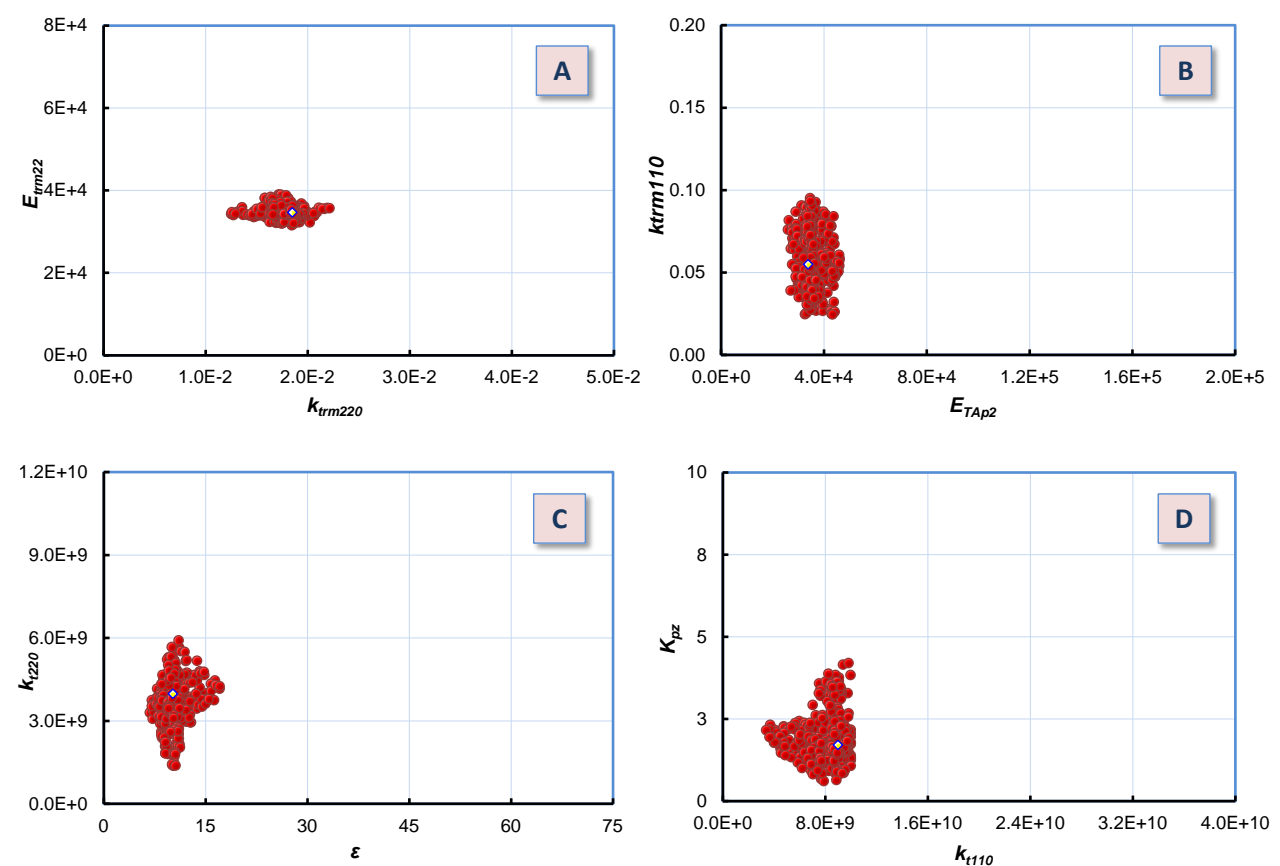

Figure 4: Joint confidence regions of A. $E_{t r m 22}$ (activation energy of transfer to styrene) vs. $k_{\text {trm } 220}$ (transfer constant of styrene), B. $k_{t r m 110}$ (transfer constant of butyle acrylate) vs. $E_{T A p 2}$ (activation energy of transfer to styrene), C. $k_{t 220}$ (termination rate coefficient of styrene) vs. $\epsilon$ (ratio of inhibition in aqueous phase and capture rate coefficients), D. $K_{p Z}$ (partition coefficient of the inhibitor between the droplets and the aqueous phase) vs. $k_{t 110}$ (termination rate coefficient of butyle acrylate).

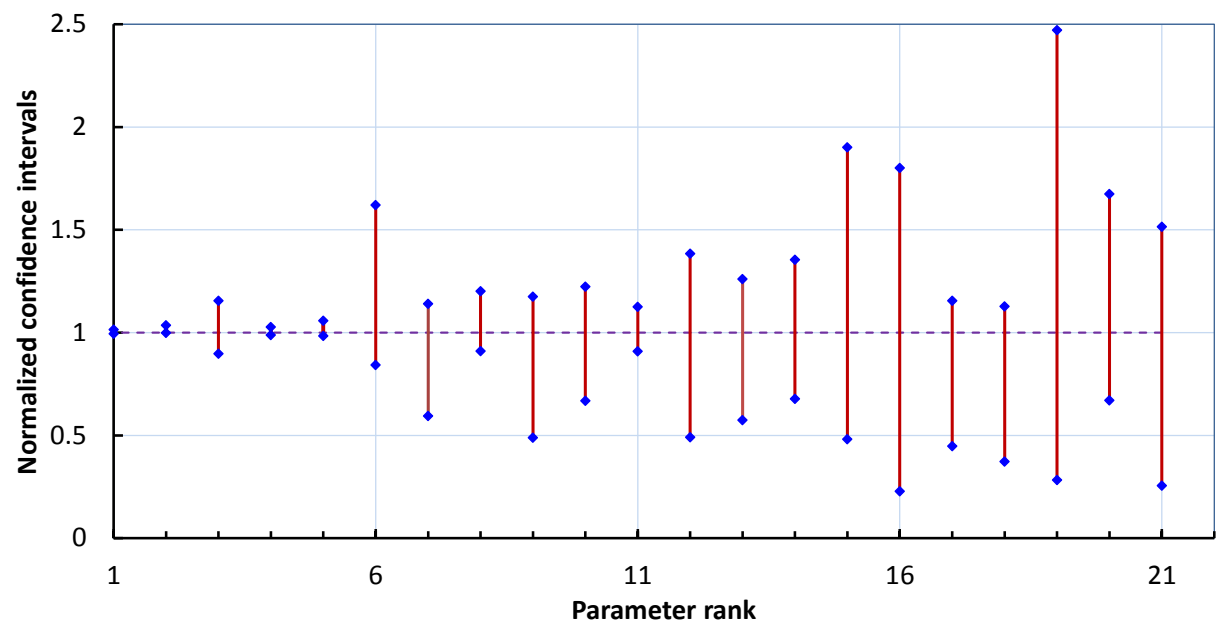

Figure 5: Normalized confidence intervals of the 21 parameters. 
which the relative accuracy is low while the estimabililty potential is high. The parameter $E_{\text {trm22 }}$ also provides a typical example of such discrepancy between the two approaches. A better match between the estimability analysis and the confidence intervals approach requires a better guess of the initial parameter values. Theoretically, this means to start from the neighborhood of the true vector of parameters or to implement the estimability analysis in a feed-back procedure. This is however beyond the scope of this paper.

\section{Conclusions}

An estimability analysis methodology based on a sequential orthogonalization procedure was implemented to distinguish the parameters potentially estimable from a set of experimental data. Thanks to this approach, 21 parameters of the most influential and the least correlated, were selected among the 49 parameters of the mathematical model of the emulsion copolymerization of styrene and butyl acrylate in the presence of a chain transfer agent.

A new method has been presented to better approximate the confidence regions of the 21 parameter estimates, as an alternative to the asymptotic confidence regions. This method can be a valuable tool to determine confidence regions more accurately, particularly in the case of non-linear systems with respect to the parameters. The relative accuracy of the 21 parameter estimates showed a good agreement with the estimability analysis. A potential improvement of the estimability analysis would be to implement it in a feed-back procedure to improve the quality of the initial guesses and provide more robust parameter ranking. Nevertheless, the parameter estimates of the 21 parameters selected by the estimbility analysis, provided a sufficient and satisfactory results in terms of model prediction capabilities.

\section{Notations}

$b^{g e} \quad$ gel coefficient of termination reaction

$b^{g l} \quad$ glass coefficient of termination reaction

$D_{p i} \quad$ diffusion coefficient of the free radicals i in the particles, $m^{2} \cdot \mathrm{s}^{-1}$

$D_{w i} \quad$ diffusion coefficient of the free radicals i in the aqueous phase, $m^{2} \cdot s^{-1}$

$E_{d} \quad$ activation energy of the initiator, $J \cdot \mathrm{mol}^{-1}$

$E_{e} \quad$ thermal expansion factor of a surfactant molecule, $K$

$E_{p 11} \quad$ activation energy of butyl acrylate propagation, $J \cdot \mathrm{mol}^{-1}$

$E_{p 22} \quad$ activation energy of styrene propagation, $J \cdot \mathrm{mol}^{-1}$

$E_{t 11} \quad$ activation energy of butyl acrylate termination reaction, $J \cdot \mathrm{mol}^{-1}$

$E_{t 22}$ activation energy of styrene termination reaction, $J \cdot \mathrm{mol}^{-1}$

$E_{t r m 11}$ activation energy of butyl acrylate monomer transfer, $J \cdot \mathrm{mol}^{-1}$

$E_{t r m 22}$ activation energy of styrene monomer transfer, $J \cdot \mathrm{mol}^{-1}$

$f \quad$ efficiency factor of initiator decomposition

$k_{d} \quad$ initiator decomposition constant, $s^{-1}$

$k_{p i j} \quad$ propagation rate coefficient of monomer $j$ with a free radical ended by $i, \mathrm{~m}^{3} \cdot \mathrm{kmol}^{-1} \cdot \mathrm{s}^{-1}$

$k_{T A, d w} \quad$ mass transfer coefficient of the CTA between droplets and aqueous phase, $m \cdot s^{-1}$

$k_{T A p i} \quad$ transfer coefficient chain transfer agent to radical $i$ in particles, $\mathrm{m}^{3} \cdot \mathrm{kmol}^{-1} \cdot \mathrm{s}^{-1}$

$k_{t i j} \quad$ termination rate coefficient (radical ended by $i$-radical ended by $j$ ), $\mathrm{m}^{3} \cdot \mathrm{kmol}^{-1} \cdot \mathrm{s}^{-1}$

$k_{\text {trmij }} \quad$ transfer to monomer rate coefficient (radical ended by $i$ - monomer $j$ ), $\mathrm{m}^{3} \cdot \mathrm{kmol}^{-1} \cdot \mathrm{s}^{-1}$ 
$k_{Z p i} \quad$ inhibition rate coefficient of radicals $i$ in the particles, $\mathrm{m}^{3} \cdot \mathrm{kmol}^{-1} \cdot \mathrm{s}^{-1}$

$k_{Z a q}$ inhibition rate coefficient in the aqueous phase, $\mathrm{m}^{3} \cdot \mathrm{kmol}^{-1} \cdot \mathrm{s}^{-1}$

$m_{d i} \quad$ equilibrium constant of free radicals ended by $i$ between aqueous and particle phases

\section{Greek letters}

$\omega_{i} \quad$ fraction of radicals ended by monomer i formed only by one monomer unit

$\delta \quad$ overall ratio of nucleation and capture rate coefficients

$\delta_{i} \quad$ ratio of nucleation and capture coefficients due to monomer unit $i$

$\delta_{m i}$ ratio of transfer resistance in aqueous phase on overall transfer resistance of free radicals ended by a monomer unit $i$

$\tau \quad$ ratio of the termination rates by disproportionation and combination

$\sigma \quad$ coefficient related to the saturation degree of the particle

$\lambda_{k} \quad k^{\text {th }}$ normalized moment of the macroradicals

\section{References}

Arzamendi, G., De la Cal, J. C., Asua, J. M., 1992. Optimal monomer addition policies for composition control of emulsion terpolymers. Angewandte Makromolekulare Chemie. 194, 47-64.

Beale, E.M.L., 1960. Confidence-regions in non-linear estimation. Journal of the Royal Statistical Society B-Statistical Methodology, 22(1), 41-88.

Benyahia, B., Latifi M. A., Fonteix, C., Pla, F., Nacef, S., 2010. Emulsion copolymerization of styrene and butyl acrylate in the presence of a chain transfer agent. Part 1: Modeling and experimentation of batch and fedbatch processes. Chemical Engineering Science, 65, 850-869.

Benyahia, B., 2009. Modélisation, expérimentation et optimisation multicritère d'un procédé de copolymérisation en émulsion en présence d'un agent de transfert de chaîne. Ph.D. thesis, National Polytechnic Institute of Lorraine, Nancy-University, France.

Benyahia, B., Latifi M. A., Fonteix, C., Pla, 2011, Multicriteria dynamic optimization of an emulsion copolymerization reactor. Computers \& Chemical Engineering, 35(12), 2886-2895.

Campi, M.C., Weyer, E., 2005. Guaranteed non-asymptotic confidence regions in system identification. Automatica, 41, 1751-1764.

Chu, Y., Huang, Z., Hahn, J., 2009. Improving prediction capabilities of complex dynamic models via parameter selection and estimation. Chemical Engineering Science, 64, 4178-4185.

DiCiccio, T.J., Efron B., 1996. Bootstrap confidence intervals. Statistic Science 11(3), 189-228.

Degenring, D., Froemel, C., Dikta, G., Takors, R., 2004. Sensitivity analysis for the reduction of complex metabolism models. J Process Control, 14(7), 729-745.

Gaia Franceschini, G., Macchietto, S., 2008, Model-based design of experiments for parameter precision: State of the art, Chemical Engineering Science, 63, 4846-4872.

Gallant, A. R., Nonlinear Statistical Models, Wiley, New York, 1987.

Gilbert, R. G. (Ed.), 1995. Emulsion polymerization. A mechanistic approach. New York: Academic Press.

Ginsburger, E., Pla, F., Fonteix, C., Hoppe, H., Massebeuf, S., Hobbes, P., Swaels, P., 2003. Modelling and simulation of batch and semi-batch emulsion copolymerization of styrene and butyl acrylate. Chemical Engineering Science. 58, 4493-4514.

Gugliotta, L. M., Arzamendi, G., Asua, J. M., 1995. monomer partition model in mathematical modeling of emulsion copolymerization systems. Journal of Applied Polymer Science. 55, 1017-1039.

Jayasankar, B.R., Ben-Zvi, A., Huang, B., 2009. Identifiability and estimability study for a dynamic solid oxide fuel cell model. Computers Chemical Engineering, 33, 484-492.

Jacquez, J.A., Greif, P., 1985. Numerical parameter identifiability and estimability: Integrating identifiability, estimability, and optimal sampling design, Mathematical Biosciences, 77(1-2), 201-227.

Joshi M, Seidel-Morgenstern A, Kremling A, 2006, Exploiting the bootstrap method for quantifying parameter confidence intervals in dynamical systems, Metabolic Engineering, 8(5), 447-455.

Lee, K.B., Lee, J.M., Park, T. S., Lee, S.H., 2010, Construction of classical confidence regions of model parameters in nonlinear regression analyses, Applied Radiation and Isotopes, 68, 1261-1265.

Li, R., Henson, M.A., Kurtz, M.J., 2004. Selection of model parameters for off-line parameter estimation. IEEE Transactions on Control Systems and Technology, 12(3), 402-412. 
Littlejohns, J.V., McAuley, K.B., Daugulis, A.J., 2010, Model for a solid-liquid stirred tank two-phase partitioning bioscrubber for the treatment of BTEX, J. Hazardous Mat., 175, 872-882.

Lund, B.F., Foss, B.A., 2008. Parameter ranking by orthogonalization - Applied to nonlinear mechanistic models. Automatica, 44, 278-281.

Martinet, F., 1992. Etude et modélisation du greffage de mélanges méthylstyrène / méthacryalyte de méthyle sur des semences de polybutadiène comme modification des mbs. Ph.D. thesis, Université Claude Bernard, Lyon 1, France.

McLean, K.A.P., McAuley, K.B., 2011. Mathematical modelling of chemical processes-obtaining the best model predictions and parameter estimates using identifiability and estimability procedures, The Canadian Journal of Chemical Engineering, DOI: 10.1002/cjce.20660.

Ngo, V.V., 2009. Modélisation du transport de l'eau et des hydrocarbures aromatiques polycycliques (HAP) dans les sols de friches industrielles, PhD Thesis, INPL, Nancy, France.

Quaiser, T., Mönnigmann, M., 2009, Systematic identifiability testing for unambiguous mechanistic modeling - application to JAK-STAT, MAP kinase, and NF- $\kappa$ B signaling pathway models, BMC Systems Biology, 3(50), 1-21.

Quiniou, S., 2009. Modélisation, simulation et analyse expérimentale du transport de matière et de chaleur dans les textiles, PhD Thesis, INPL, Nancy, France.

Rawlings, J. B., Ray, W. H., 1988. The modeling of batch and continuous emulsion polymerization reactors. part i. model formulation and sensitivity to parameters. Polymer Engineering and Science. 28(5), 237-255.

Rooney, W.C., and Biegler, L.T., 2001. Design for Model Parameter Uncertainty Using Nonlinear Confidence Regions AIChE Journal, 7(8), 1794-1804.

Salazar, A., Gugliotta, L. M., Vega, J. R., Meira, G. R., 1998. Molecular weight control in a starved emulsion polymerization of styrene. Industrial and Engineering Chemistry Research. 37(9), 3582-3591.

Schwaab, M., Biscaia, E.C.Jr., Monteiro, J.L., Pinto, J.C., 2008. Nonlinear parameter estimation through particle swarm optimization, Chemical Engineering Science, 63(6), 1542-1552.

Seber, G., and C. Wild, Nonlinear Regression, Wiley, New York, 1989.

Sgard, A., 2000. Modélisation de procédés discontinus et semi-continus de polymérisation en émulsion. Ph.D. thesis, INPL, Nancy, France.

Snuparek, J.J. and Kleckova, Z., 1984. Some factors affecting the particle growth in semicontinuous emulsion polymerization of acrylic monomers, Journal of Applied Polymer Science, 29, 1-11.

Surisetty, K., Siegler, H.D.H., McCaffrey, W.C., Ben-Zvi, A., 2010. Model re-parameterization and output prediction for a bioreactor system, Chemical Engineering Science, 65, 4535-4547.

Turanyi T., 1990. Reduction of large reaction mechanisms, New J Chemistry, 14, 795-803.

Vajda, S., Rabitz, H., Walter, E., Lecourtier, Y., 1989. Qualitative and quantitative identifiability analysis of nonlinear chemical kinetic models, Chemical Engineering Communications, 83, 191-219.

van Boekel, M. A. J.S 1996, Statistical aspects of kinetic modeling for food science problems. Journal of Food Science, 61(3), 477-489.

Varziri, M. S., McAuley, K.B. McLellan, P. J., 2008. Parameter Estimation in Continuous-Time Dynamic Models in the Presence of Unmeasured States and Nonstationary Disturbances. Industrial Engineering Chemical Research, 47, 380-393.

Varziri, M. S., McAuley, K.B. McLellan, P. J., 2008. Parameter and State Estimation in Nonlinear Stochastic ContinuousTime Dynamic Models With Unknown Disturbance Intensity, The Canadian journal of Chemical Engineering, 86, 828-837.

Velez-Reyes, M. and Verghese, G.C., 1995. Subset selection in identification, and application to speed and parameter estimation for induction machines, Proc. 4th IEEE Conf. Control Appl., 991-997.

Walter, E., Pronzato, L., 1994. Identification of parametric models from experimental data. Springer, Berlin.

Weijers, S. R., Vanrolleghem, P.A., 1997. Procedure for selecting best identifiable parameters in calibrating activated sludge model no. 1 to full-scale plant data, Water Science and Technology, 36(5), 69-79.

Wu, S., McLean, K. A.P., Harris, T.J., McAuley, K.B., 2011. Selection of optimal parameter set using estimability analysis and MSE-based model-selection criterion, International J Advanced Mechatronic Systems,3(3), $188-197$.

Yao, K.Z., Shaw, B.M., Kou, B., McAuley, K.B., Bacon, D.W., 2003. Modelling ethylene/butene copolymerization with multi catalyst: parameter estimability and experimental design, Polymer Reaction Engineering., 11(3), 563-588.

Yeh, A.B., Singh, K., 1997. Balanced Confidence Regions Based on Tukey's Depth and the Bootstrap, Journal of the Royal Statistical Society B-Statistical Methodology, 59(3), 639-652. 